\title{
Unerupted Maxillary Central Incisor Associated with Compound Composite Odontoma: A Case Report
}

Kamal Malhotra', Ritu Namdev², Monika Rohilla ${ }^{3}$, Samir Dutta ${ }^{4}$

\begin{abstract}
Odontoma is the most common type of benign odontogenic tumor, and often causes disturbances in the eruption of its associated tooth. This case report documents a twelve -year-old-child with a compound odontoma located in the anterior maxilla, which caused the impaction of the maxillary central incisor. Spontaneous eruption of the involved tooth occurred once the odontoma was surgically removed.
\end{abstract}

Keywords: Odontoma, Impacted, Compound, Composite, Eruption

${ }^{1}$ Post Graduate Student,

Department of Pedodontics,

Government Dental College,

Rohtak, Haryana, India

${ }^{2}$ MDS,

Assistant Professor,

Department of Pedodontics

Government Dental College,

Rohtak, Haryana, India

${ }^{3}$ MDS,

Assistant Professor,

Department of Pedodontics

Government Dental College,

Rohtak, Haryana, India

${ }^{4} \mathrm{MDS}$,

Senior Professor and Head

Department of Pedodontics

Government Dental College,

Rohtak, Haryana, India

\section{Contact Autbor}

\author{
Dr. Ritu Namdev \\ ritunamdev@ rediffmail.com
}

J Oral Health Comm Dent 2012;6(1)43-46

\section{INTRODUCTION}

In clinical setting, dentists often encounter the problem of tooth impaction, which has been defined as a situation where a tooth fails to erupt into a normal functional position by the expected time. Unerupted teeth are seen more commonly in permanent dentition and are relatively common in early mixed dentition. Impacted teeth can cause serious dental and aesthetic difficulties as well as psychological problems especially in anterior regions. Although the impacted maxillary incisor occurs less frequently than the maxillary canine, it is of concern to parents during the early mixed dentition stage because of noneruption of the tooth. Maxillary central incisor impactions occur infrequently; their origins include various local causes, such as odontoma, supernumerary teeth, space loss, and disturbances in the eruption path, also trauma and apical follicular cysts. Odontomas are considered to be developmental anomalies resulting from the growth of completely differentiated epithelial and mesenchymal cells that give rise to ameloblasts and odontoblasts. These tumors are basically formed of enamel and dentin but they can also have variables amounts of cementum and pulp tissue. During the development of the tumor, enamel and dentin can be deposited in such a way that the resulting structures show an anatomic similarity to normal teeth, in which case the lesion is classified as a compound odontoma. However, when the dental tissues form a simple irregular mass occurring in a disorderly pattern, it is described as a complex odontoma. Compound odontomas appear more frequently than complex odontomas. The majority of odontomas which are located in the anterior region of the maxilla are compound, while the great majority of odontomas located in the posterior areas, especially in the mandible, are complex odontomas (1-3). The etiology of odontoma is unknown, although local trauma, infection and genetic factors have been suggested (4). In general they are asymptomatic, have slow growth, and seldom exceed the size of a tooth, but when large can cause expansion of the cortical bone. Odontomas may be diagnosed at any age but they are usually detected during the first two decades of life. Despite the fact that tumour is not aggressive and has a limited growth potential, early diagnosis is very important so that complications in dental arch development and occlusion, including aesthetics, are avoided. Many times odontomas are found associated with 


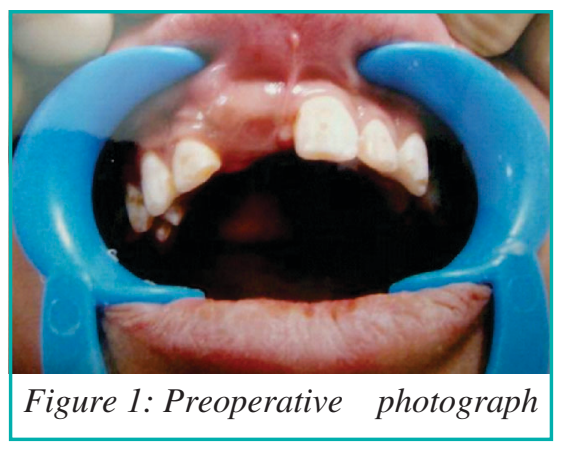

unerupted teeth. The canines, followed by upper central incisors and third molars, are the most frequent teeth impacted by odontomas. Generally these malformations are intraosseous, but occasionally they may erupt into the oral cavity.

A developing odontoma can be discovered by routine radiography, but may cause difficulty in identification due to lack of calcification $(1,5)$. The differential diagnosis of odontoma is made by roentgen graphic examination, followed by surgical removal and histological examination with osseous recontouring. Recurrences are uncommon (6).

\section{CASE REPORT}

A 12 year old male patient reported to the Dept. of Pedodontic and Preventive Dentistry, Govt Dental College \& Hospital, Rohtak with a chief complaint of unerupted anterior tooth. The family and medical histories were insignificant. No history of trauma to face or mouth was recalled. Extraoral examination was non contributory. Intraoral examination revealed unerupted 11 without any swelling or inflammation of the muoosa while the contralateral tooth had already erupted (Figure 1). An intra-oral periapical, occlusal and

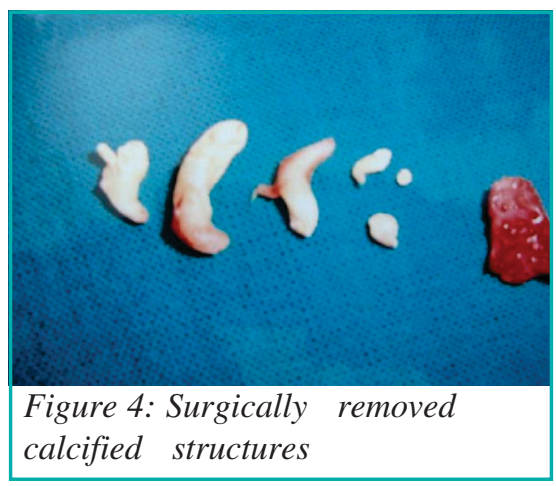

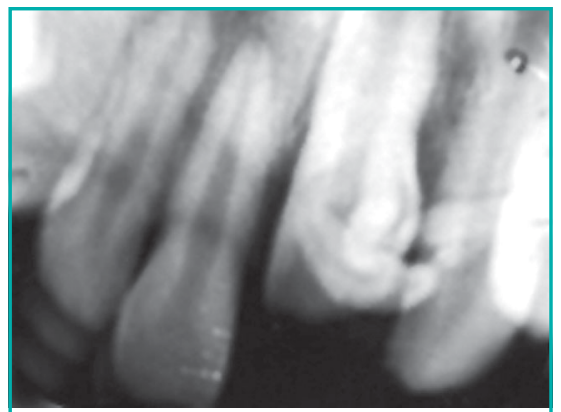

Figure 2: Intraoral periapical radiograph showing calcified structures

panoramic radiographs revealed the presence of a radio opaque mass in 11 region thereby obstructing the eruption of 11(Figure 2). On the basis of clinical and radiographic findings, it was provisionally diagnosed as an odontoma. Surgical removal of the odontoma under local anaesthesia was planned. A mucoperiosteal flap on the labial surface from the right lateral incisor to the left lateral incisor was reflected. The layer of bone overlying the labial surface was removed and the calcified mass was exposed (Figure 3). Surgical removal was performed and a number of calcified small structures looking like teeth were found in it and removed without disturbing the underlying tooth and sent for histopathological examination (Figure 4). Curettage was done in the area and the area was debrided of any remnants. In order to ensure that no denticles remained, radiographs were taken before closure of the flap. After haemostasis, the area was irrigated with saline solution and the mucoperiosteal flap was sutured back in position. Postoperative instructions specifically related to the maintenance of an appropriate oral hy-

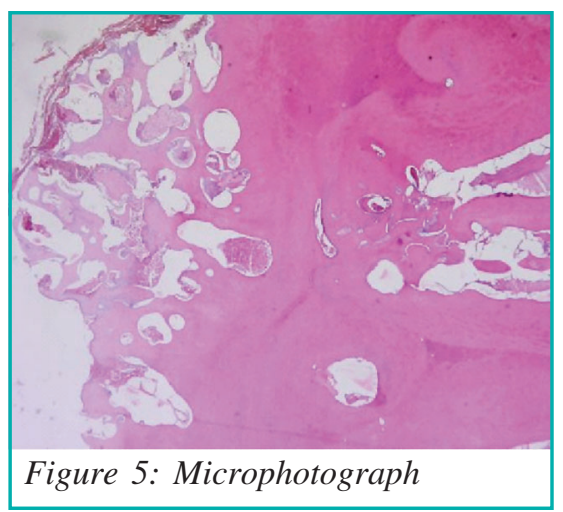

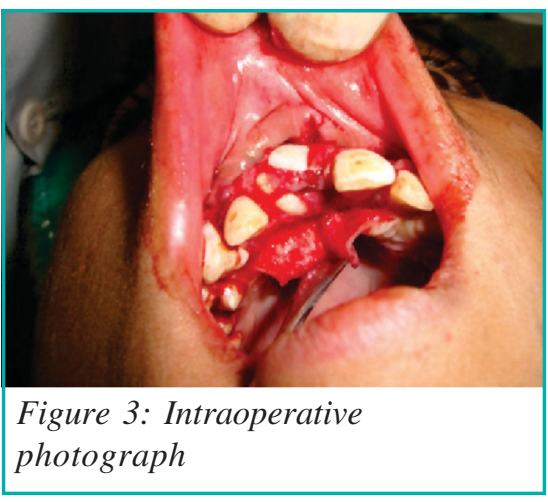

giene, ingestion of cold and soft meals, refraining from physical exercise during 48 hours, and management of pain were given to the patient's mother. Healing was uneventful and on the seventh day after the surgery, the sutures were removed. Patient was advised a review appointment once in 3 months to assess the eruption of unerupted tooth 11 and to examine for the recurrence of the odontoma.

The protocol that is followed was clinical and radiographic -examination every six months and clinical examination alone in the other visits. The decalcified section showed dentin in cross section and longitudinal sections. The dentin appears to have normal tubular pattern. Strands of connective tissue, empty spaces of pulp chamber were also seen (Figure 5). These findings were suggestive of compound composite odontoma. It was seen that the tooth 11 had more than two third of root formation and it was noted during consecutive follow ups that there was a significant change in its position. Patient was followed up for more than a year and the permanent right central incisor was observed to move down along the path of eruption without any evidence of recurrence of the bony mass. After 12 months it finally reached occlusal plane without need for orthodontic traction (Figure 6-8).

\section{DISCUSSION}

Odontomas are relatively common odontogenic lesions, generally asymptomatic, and are rarely diagnosed before the second decade of life. They frequently lead to impaction or delayed eruption of permanent teeth. Odontomas have been associated with trauma during primary dentition, as 


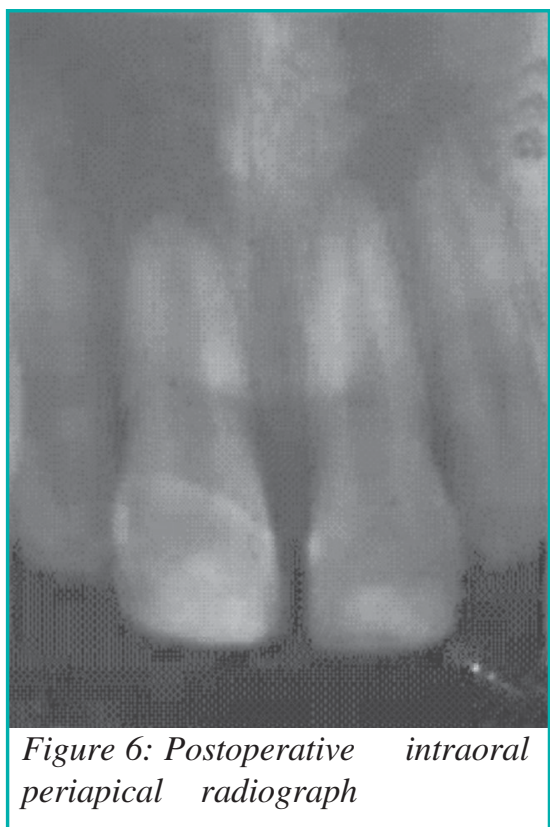

well as with inflammatory and infectious processes, hereditary anomalies (Gardner's syndrome, Hermann's syndrome), odontoblastic hyperactivity and alterations in the genetic components responsible for controlling dental development(7). According to the latest classification of the World Health Organization (WHO,2005), two types of odontomas can be found: complex odontomas and compound odontomas - the latter being twice as common as the former. Compound odontomas are usually located in the anterior sector of the upper maxilla, over the crowns of unerupted teeth, or between the roots of erupted teeth. The lesions are usually unilocular and contain multiple radiopaque, miniature tooth-like structures known as denticles. There are three main types of compound composite odontomal. Denticular type - composed of two or more

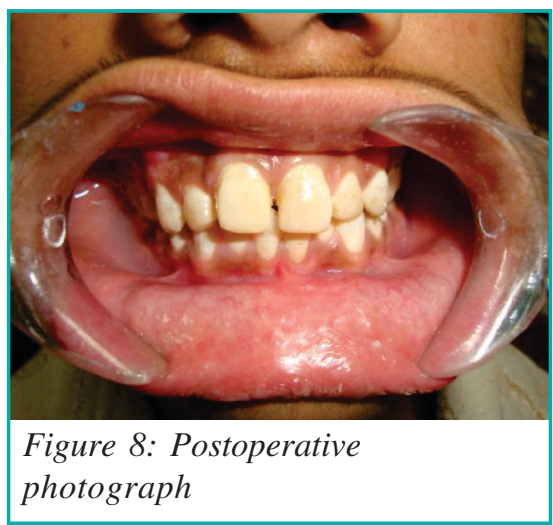

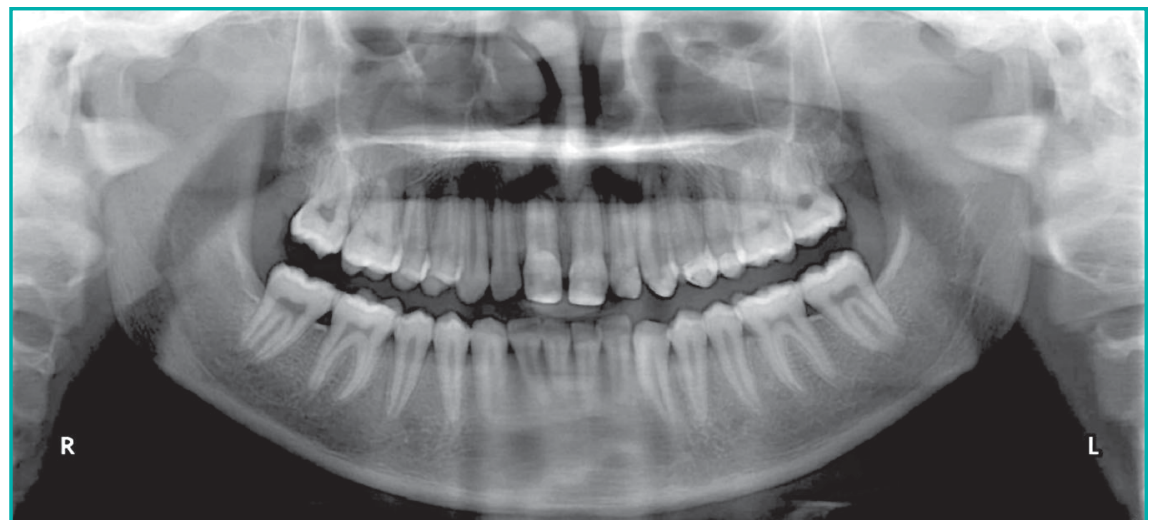

Figure 7: Postoperative orthomopantogram

separate denticles, each having a crown and a root or epithelial sheath of Hertwig with a distribution of dental hard tissues comparable to that found in a tooth. Particulate type - composed of two or more separate masses or particles bearing no macroscopic resemblance to a tooth and consisting of hard dental tissues abnormally arranged. Denticulo particulate type - in this type denticles and conglomerate masses or particles are present side by side. Complex odontomas in turn are found in the posterior mandibular sector, over impacted teeth, and can reach several centimeters in size. Radiologically, these lesions manifest as a radiopaque solid mass with occasional nodular elements, and surrounded by a fine radiotransparent zone. The lesions are unilocular and are separated from the normal bone by a well defined corticalization line. No individual tooth-like structures are seen. Epidemiologically; odontomas are the most frequent odontogenic tumors, with an incidence of $22-67 \%$ of all maxillary tumors. The lesions are more often found in children and adolescents, and there are scant differences between males and females. Clinically, these are asymptomatic lesions often associated to alterations in permanent or temporary tooth eruption. The diagnosis is usually established on occasion of routine radiological studies (panoramic and/or intraoral X-rays), or on evaluating the cause of delayed tooth eruption. The treatment of choice is surgical removal of the odontoma, followed by histological analysis. Clinical experience suggests and the dental literature supports that an individualized radiographic examination of any pediatric patient that presents clinical evidence of delayed permanent tooth eruption or temporary tooth displacement with or without a history of previous dental trauma should be performed. As was demonstrated by this report, early diagnosis of odontomas allows adoption of a less complex and less expensive treatment and ensures better prognosis (1). If an odontoma is found to be attached to an adjacent tooth, a periapical radiograph during the surgical phase is advisable since surgical sites can be obscured and the attachment may extend farther than first imagined. The single additional periapical radiograph should prove preventive, i.e., preventing additional surgery and/or extended orthodontic treatment (6). In the case of odontomas associated to impacted teeth, the latter should be preserved in wait of spontaneous eruption, or alternatively fenestration followed by orthodontic traction are indicated (8). The location of the impacted tooth was classified into three patterns: 1) The impacted tooth was under the odontoma. 2) The impacted tooth was next to the odontoma. 3) The impacted tooth migrated to an ectopic location. There is no general agreement on the best management approach for impacted teeth associated to odontomas. The treatment options comprise surgical extraction, fenestration and posterior orthodontic traction, or simple observation with periodic clinical and radiological controls, to evaluate the course of these teeth(9). In this case, the lesion was surgically removed, and the impacted central incisors finally reached the occlusal plane after 12 months without 
the need for orthodontic traction. This was probably attributable to the preserved eruptive capacity of impacted teeth in young patients. Morning, in a study of 42 odontomas associated to impacted teeth, found $44 \%$ of these teeth to erupt spontaneously after removal of the odontoma(10). The rest of the teeth required fenestration to complete eruption into the mouth. Hisatomi et al. reported that such impacted teeth tend to erupt, regardless of the degree of root formation after extirpation of the odontoma interfering with tooth eruption, although some teeth showed infraversion and/or crowding(11). Thus, the treatment of choice for these impacted teeth associated to odontomas appears to be removal of the lesion with preservation of the impacted tooth. The latter in turn require clinical and radiological followup for at least one year. If no changes in the position of the tooth are seen during this period, fenestration followed by orthodontic traction is indicated (12). Extraction should be carried out in those cases where the tooth is ectopic or heterotopic, with morphological alterations, or presenting cystic lesions. In general, if the root of the impacted tooth is still developing, the tooth may erupt normally; but, once the root apex has closed, the tooth has lost its potential to erupt. Interestingly, orthodontic therapy is not usually necessary and is not applied to improve the malocclusion caused by compound odontoma after extirpation of the tumour. The reason is that most odontomas are very small, and the influence of the tumor on occlusion might be improved without orthodontic therapy. If detected at earlier stages, interceptive treatment can be provided effectively enhancing esthetics, functions and structural balance in developing dentition. Early removal of the cause of eruption disturbances is important in the developing dental arch. Ameloblastic fibro-odontomas and odontoameloblastomas show a great resemblance to common odontomas, especially in the radiographic examination. Therefore, it has been suggested that all specimens should be sent to an oral pathologists for microscopic examination. The histological examination of odontomas often shows the presence of enamel matrix, dentin, pulp tissue, and cementum that can, but need not, exhibit a normal relationship. Compound odontomas are formed by tooth-like structures which resemble pulp tissue in the central portion surrounded by a dentin shell and partially covered by enamel. Complex odontomas are conglomerates without orientation of dentin, enamel, enamel matrix, cementum, and areas of pulp tissue. The capsule of connective tissue that surrounds an odontoma is similar to the follicle that covers a normal tooth. Literature suggested that odontoma once enucleated usually does not recur but in young children a close monitoring is necessary. An exceptional circumstance is spontaneous eruption of an odontoma into the oral cavity, i.e., exposure of the tumor through the oral mucosa. This situation can cause pain, inflammation of the adjacent soft tissues, or infection associated with suppuration (13).

\section{REFERENCES}

1. Branca Heloísa de Oliveira, Vera Campos, Sonia Marçal. Compound odontoma diagnosis and treatment:three case reports. Pediatric Dentistry 2001;23(2): 151-57.

2. Owens BM, Schuman NJ, Mincer HH, Turner JE, Oliver FM. Dental odontomas: a retrospective study of 104 cases. J Clin Pediatr Dent 1997;21:261-64.

3. Katz R. An analysis of compound and complex odontomas. J Dentist Child 1989;56:445-49.

4. Das UM, Nagarathna Arathi. Unerupted maxillary primary canine associated with compound composite odontoma : a case report. Journal of Indian Society of Pedodontics and Preventive Dentistry 2002;20( 3):98-101.

5. Brunetto AR, Turley PK, Brunetto AP, et al. Impaction of a primary maxillary canine by an odontoma: surgical and orthodontic management. Pediatr Dent 1991; 13:301302.

6. Franka C. Dubbelman-Falcon, Edward M. Sonnenberg, et al. Odontoma attached to an unerupted maxillary central incisor. Pediatric Dentistry 1982;4(4):338-40.

7. Ragalli CC, Ferreria JL, Blasco F. Large erupting complex odontoma. Int $\mathrm{J}$ Oral Maxillofac Surg 2000;29(5):373-74.

8. Serra-Serra G, Berini-Aytés L, GayEscoda C. Erupted odontomas: A report of three cases and review of the literature. Med Oral Patol Oral Cir Bucal 2009; 14(6):299-303.

9. Frank C. Treatment options for impacted teeth. J Am Dent Assoc 2000; 131:62332

10. Morning P. Impacted teeth in relation to odontomas. Int J Oral Surg 1980;9:8191.

11. Hisatomi M, Asaumi J, Konouchi $\mathrm{H}$, et al. A case of complex odontoma associated with an impacted lower deciduous second molar and analysis of the 107 odontomas. Oral Dis 2002;8:100-05.

12. Oliver RG, Hodges CGL. Delayed eruption of a maxillary central incisor associated with an odontome: report of case. $A S D C$ J Dent Child 1988;55:368-71.

13. Litonjua L, Suresh L, Valderrama L, Neiders M. Erupted complex odontoma: A case report and literature review. Gen Dent 2004;52:248-51. 